\title{
Comparative Evaluation of Expansion of gutta-percha in contact with two different powder/liquid ratios of Zinc Oxide Eugenol sealer - An in vitro study.
}

\author{
${ }^{1}$ Dr.Amol Bodhankar, ${ }^{2}$ Dr.Manoj Chandak, ${ }^{3}$ Dr.N U Manwar, \\ ${ }^{4}$ Dr.Pradnya Nikhade, ${ }^{5}$ Dr.Ajay Saxena \\ ${ }^{1}$ (Postgraduate Student, Department of Conservative Dentistry and Endodontics, Sharad Pawar Dental College \\ and Hospital, Sawangi (Meghe) Wardha, Maharashtra. Pin code - 442004) \\ ${ }_{2}^{2}$ (Professor and Head of the Department, Department of Conservative Dentistry and Endodontics, Sharad \\ Pawar Dental College and Hospital, Sawangi (Meghe) Wardha,Maharashtra. Pin code - 442004) \\ ${ }^{3}$ (Professor, Department of Conservative Dentistry and Endodontics, Sharad Pawar Dental College and \\ Hospital, Sawangi (Meghe) Wardha, Maharashtra. Pin code - 442004) \\ ${ }^{4}$ (Professor Department of Conservative Dentistry and Endodontics, Sharad Pawar Dental College and \\ Hospital, Sawangi (Meghe) Wardha, Maharashtra. Pin code - 442004) \\ ${ }_{5}^{5}$ (Associate Professor Department of Conservative Dentistry and Endodontics, Sharad Pawar Dental College \\ and Hospital, Sawangi (Meghe) Wardha, Maharashtra. Pin code - 442004)
}

\begin{abstract}
The aim of root canal obturation is to create a "impermeable seal" to prevent theingress of bacteria and their toxins. To achieve this goal, disinfection of the canalsystem is accomplished by thorough chemomechanical debridement of the pulpaltissue, dentin debris, microorganisms, and the use of one or more disinfectants.

Obturation of the canal system has historically been achieved with gutta-percha and an appropriate sealer to obtain a tight seal. There has been an ampleamount of research determining the dimensional changes and expansion of rootcanal sealer materials, but none have directly addressed the effect of dimensionalchanges on the gutta-percha cones
\end{abstract}

The aim of the study was comparative evaluation of gutta-percha in contact with two different ratios of zinc Oxide eugnol sealer. The purpose of the study was to evaluate Expansion of gutta-percha in contact with Zinc Oxide Eugenol sealer Powder/Liquid Ratio of 1:1 and 1:5 after 1 day, 7 days and 30 days.

Result : 30 days after the obturation, both ratios showed equal amount of expansion which was statistically insignificant $(p<0.05)$

Keywords : Gutta-percha, Zinc Oxide Eugenol sealer, Spiral computed tomography.

\section{Introduction :}

Most root canal sealers shrink duringsetting and dissolve with time (1-3). Orstavik et al. found that zincoxide- eugenol- based sealers shrank $0.3 \%-1 \%$ over time. Shrinkage and dissolutionof sealer result in formation of voids and have the potential to promoteleakage and eventual endodontic failure (4). Some studies have shown an interactionbetween gutta-percha and certain sealers (5-7). Wu et al. found that whencanals were obturated with just sealer alone, there was a dramatic increase inleakage over time as a result of sealer dissolution. They also demonstrated that withand without sealer gutta-percha had a reduction in leakage over time, whichindicated an increase in volume, with the expansion of gutta-percha compensatingfor sealer dissolution, resulting in decreased leakage of the canal system.

Eugenol has successfully been used in dentistry for more than 125 years and has been incorporated in many of today's endodontic sealers. Eugenol has many therapeutic effects including antimicrobial and anesthetic properties. Eugenol $(\mathrm{C} 10 \mathrm{H} 12 \mathrm{O} 2)$ is an allyl chain-substituted guaiacol extracted from certain essentialoils, especially from clove oil. It is a weak acid with a pKa of 10.4 and occurs as hydrogen bonded dimers. The first zinc oxide- eugenol sealer was popularized by Rickert and Dixon in 1933 and has been the standard of the profession for many years (8)

\section{Selection of samples:}

\section{Materials and Methods :}

A total thirty human mandibular permanent premolars with complete root formation which were extracted for orthodontic purposes were selected for the study.

Storage of the samples: 
Immediately after extraction, the teeth were kept in hydrogen peroxide solution washed under running tap water for 15 minutes each; the external surfaces of all teeth were cleaned with periodontal curettes to removes the debris and soft tissues. Later they were cleaned with pumice and stored in normal saline at $40^{\circ} \mathrm{C}$ until use. Collection, storage, sterilization, and handling of extracted teeth followed the Occupational Safety and Health Administration guidelines and regulations.

\section{Preparation of samples:}

\section{Methodology:}

Teeth were decoronated at cementoenamel junction (CEJ) to standardize the root length in all the groups. A \#10 K-file was introduced until the tip was just visible at the foramen of the root. The working length was determined by subtracting $0.5 \mathrm{~mm}$ from the measured length. Cleaning and shaping were performed in all teeth initially with hand K-files up to 25 followed by HEROshapers upto 26/06. Each preparation was rinsed with saline for 20 seconds. Before obturation, root canals were dried with a paper point. Obturation was done by using gutta-percha points (25/04) (Dentsply Maillefer, Ballaigues, Switzerland)

Then the teeth were randomly divided into three groups.

Group A- Consisted of 10 samples, obturated with Gutta-percha in contact with zinc-Oxide eugenol Sealer with powder/ liquid Ratio of 1:1 (ie $1 \mathrm{gm}: 1 \mathrm{ml}$ ) for 5 seconds

GroupB- Consisted of 10 samples, obturated with Gutta-percha in contact with zinc-Oxide eugenol Sealer, powder/ liquid Ratio of 1:5 (ie $1 \mathrm{gm}: 5 \mathrm{ml}$ ) for 5 seconds.

Group C- Consisted of 10 samples, obturated with Gutta-percha without using sealer.

All the samples were subjected to spiral CT scan, 1 day, 7 days and 30 days after the day of obturation. Specimens were scanned by using a Siemens Sensation 4 model of Spiral CT (SiemensAG, Munich, Germany). They were then viewed under high resolution, both cross-sectionally and longitudinally, with a constant thickness of $0.5 \mathrm{~mm} / \mathrm{slice}$ and a constant spiral or table speed of 0.5 and $120 \mathrm{kVp}$. The area of prepared root canal in each slice was measured from CEJ to the apex of the root. The volume of root canal in each tooth was estimated with the aid of Syngo software.

\section{(III) Results :}

\section{Graph 1 : Representing expansion of gutta-percha for Group A}

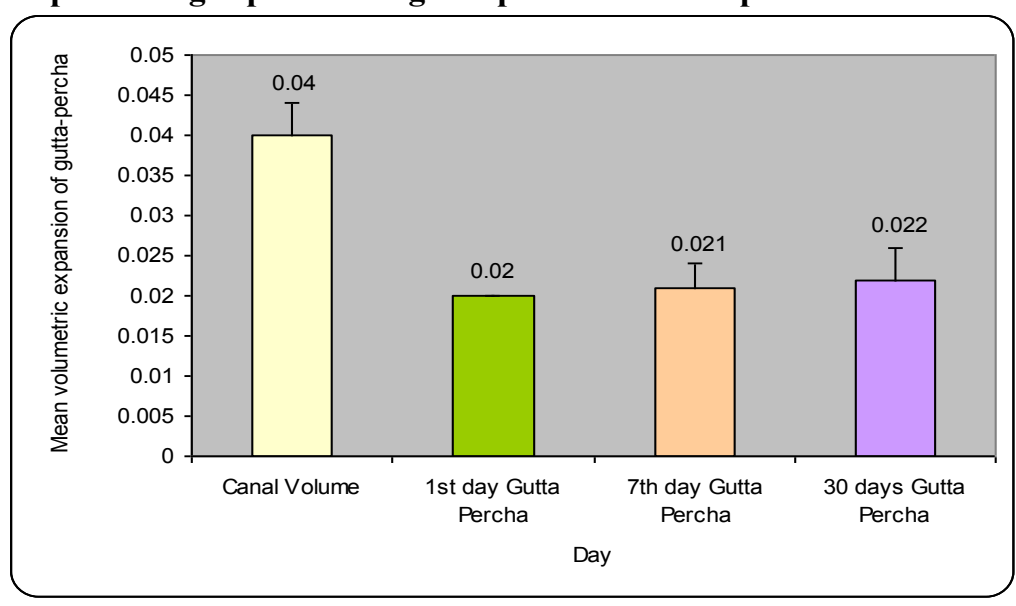


Graph 2 : Representing expansion of gutta-percha for Group B

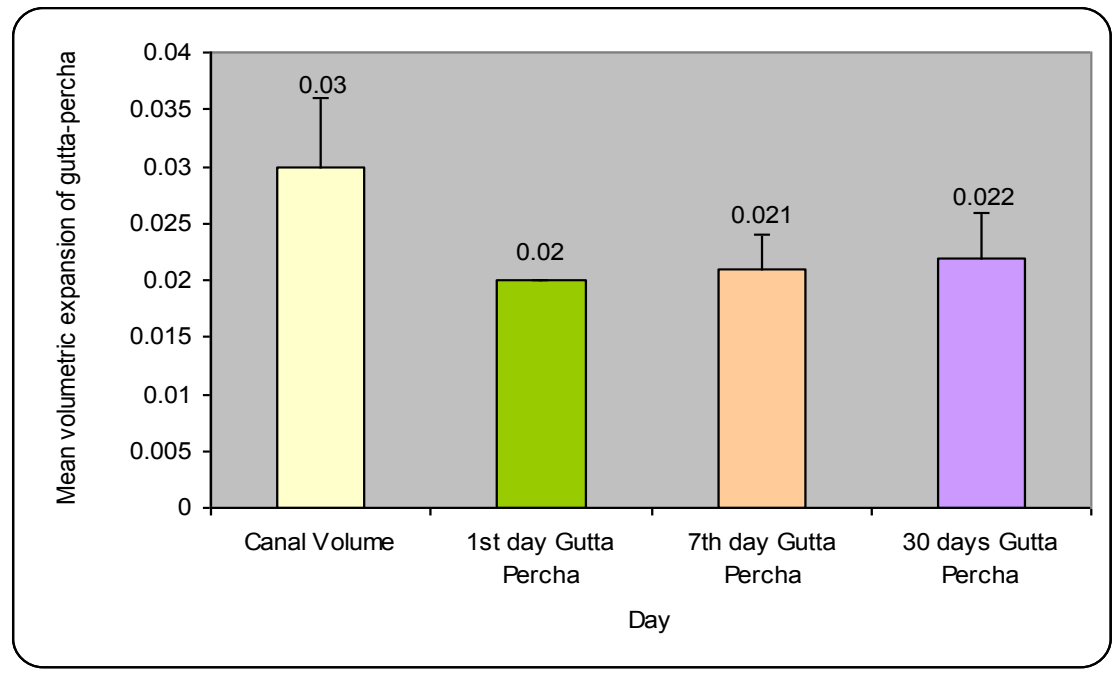

Graph 3 : Representing expansion of gutta-percha for Group C

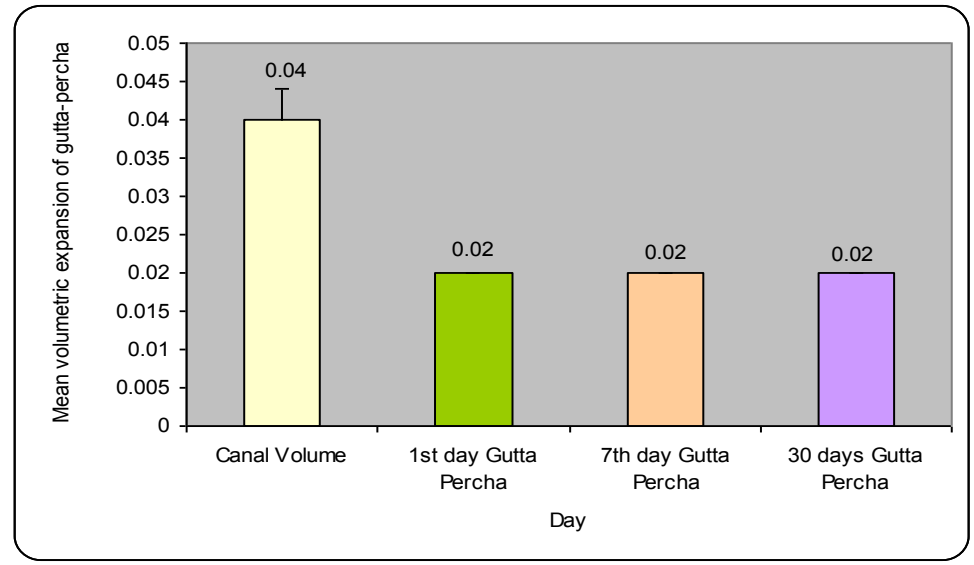

Results showed that both the groups, Group A (powder/liquid Ratio 1:1) and Group B (powder/liquid ratio 1:5) exhibited no expansion of gutta-percha 1 day after the obturation.

Group A and Group B showed slight expansion of gutta-percha, 7 days after the obturation (from $0.020 \mathrm{~cm}^{3}$ to $0.021 \mathrm{~cm}^{3}$ )

Group A and Group B showed slightly more but equal amount of expansion of gutta-percha, 30 days after the obturation. (from original volume of gutta-percha ie $0.020 \mathrm{~cm}^{3}$ to $0.022 \mathrm{~cm}^{3}$ ). However, when sealer was not used for obturation ie Group C showed no expansion of gutta percha, even 30 days after the obturation.

\section{Discussion.}

Zinc Oxide Eugenol sealer was used in study as Unlike resin-based sealers, setting reaction of Zinc Oxide Eugenol-based sealers is a chelation reaction occurring between eugenol and the zinc ion of the zinc oxide. This reaction might also occur with the zinc oxide phase of gutta-percha along with the calcium ions of dentin. This might explain the decreased setting shrinkage associated with the ZOE-based sealers.

Kardon et al(2003)have shown that urethane dimethacrylate-based resin sealers can be used for single cone technique and also stated that dimensional changes and extended setting shrinkage of root canal sealers, in particular methacrylate-based resin sealers when compared with traditional Zinc Oxide Eugenol-based sealers. Michaud et al(2008)have shown that when the butt ends of gutta-percha cones were placed in 3-mm-deep eugenol, they expanded dramatically in size. volumetric expansion of gutta-percha occurred in contact with eugenol during a 30 -day period, which was almost $135.35 \%$, and also was substantiated through a pilot study done earlier, which showed a remarkable increase in the gutta-percha dimensions when placed in eugenol that continued even after 4.5 years. Study showed that dimensional changes of gutta-percha would occur when in contact with eugenol(9). Nair MK and Nair UP have shown that Spiral Computed Tomography is a 
noninvasive technique that gives a three-dimensional interpretation at various levels. It also avoids loss of material and yields reproducible results, and the specimens can be used for further research. The percentage of expansion at a specific location can be determined accurately. Recent studies have proved that SCT provides accurate volumetric analysis of root fillings and remaining remnants of root fillings (10).Wilson AD and Bachelor RF (1970) stated that increased powder/liquid ratio of Zinc Oxide

Eugenol sealer reduced the amount of released eugenol at 1 month period, which can be considered to be a cause for less Gutta-percha expansion in Group A(Powder/Liquid Ratio 1:1)(11).Camps et al (2004), reported that the higher the powder/ liquid ratio, the lower the eugenol release, which might be due to the supplementary zinc oxide particles binding to eugenol as fast as it is released; thus only the outer surface of sealer is prone to eugenol release. Therefore, a ratio of 1:1 (Powder/Liquid) sealer concentration may have shown less expansion. However, Pommel L, Bukiet F(2004), stated that increasing the eugenol content may not cause expansion as much as when eugenol is moderately increased. Authors stated it could be due to insufficient amount of contact time of gutta-percha with Zinc Oxide Eugenol sealer, which is very thin in consistency so leading to decreases contact time of gutta-percha with the eugenol which can be considered to be the cause of insignificant expansion of gutta-percha when 1:55 powder/liquid ratio was used(12).

\section{Limitations of the study :}

- The study was an in -vitro study, the results may vary when performed in clinical conditions.

- The study did not evaluate the expansion of gutta-percha with other sealers.

\section{Conclusion :}

Under the limitations of the study, based on the results obtained and discussed the following conclusions were drawn from the present study

1) Higher the powder/liquid ratio, lesser will be the expansion of gutta-percha due to decreased availability of eugenol.

2) If Eugenol content is increased too much (ie 1:5), then too there was less expansion of gutta-percha observed due to increase in consistency leading to decreased contact time of gutta-percha to the eugenol.

\section{Acknowledgement :}

The authors would like to extend their appreciation to Dr.Prashant Nimbalkar, Dr.Nilay Nimbalkar, Dr.Parimal Fuke, Precision Scan and Research Centre Pvt. Ltd, Dhantoli, Nagpur.

\section{Bibliography :}

[1]. Orstavik D. Weight loss of endodontic sealers, cements and pastes in water. Scand J Dent Res 1983;91:316 -9

[2]. Tronstad L, Barnett F, Flax M. Solubility and biocompatibility of calcium hydroxide containing root canal sealers. Endod Dent Traumatol 1988;4:152-9.

[3]. Kontakiotis EG, Wu MK, Wesselink PR. Effect of sealer thickness on long-term sealing ability: a 2-year follow-up study. Int Endod J 1997;30:307-12.

[4]. Orstavik D, Nordahl I, Tibballs JE. Dimensional change following setting of root canal sealer materials. Dent Mater 2001;6:512-9.

[5]. 5)Wu M-K, Wesselink PR, Boersma J. A 1-year follow-up study on leakage of four root canal sealers at different thicknesses. Int Endod J 1995;28:185-9.

[6]. Wu MK, Fan B, Wesselink PR. Diminished leakage along root canals filled with gutta percha without sealer overtime: a laboratory study. Int Endod J 2000; 33:121-5.

[7]. Tagger M, Greenberg B, Sela G. Interaction between sealers and gutta-percha cones. J Endod 2003;29:835-7

[8]. Grossman L. An improved root canal cement. J Am Dent Assoc 1958;56:381.

[9]. A. Michaud, John Burgess, Robert D. Barfield, DenizCakir, Sandre F. McNeal, and Paul D. Eleazer, Volumetric Expansion of GuttaPercha in Contact With Eugenol J Endod 2008;34:1528-1532.

[10]. Nair MK, Nair UP. Digital and advanced imaging in endodontics: a review. J Endod 2007;33:1

[11]. Wilson AD, Batchelor RF. Zinc oxide - eugenol cements II: study of erosion and disintegration. J Dent Res

[12]. Camps J, Pommel L, Bukiet F, About I Influence of the powder/liquid ratio on the properties of zinc-oxide eugenol based root canal sealers. Dent Mater 2004;20: 915-23 1970;49: 593-8. 